\title{
Relationship of menopausal status and climacteric symptoms to sleep in women undergoing chemotherapy
}

\author{
Michelle B. Rissling • Lianqi Liu • Loki Natarajan • \\ Feng He $\cdot$ Sonia Ancoli-Israel
}

Received: 4 May 2009 / Accepted: 11 May 2010 / Published online: 29 May 2010

(C) The Author(s) 2010. This article is published with open access at Springerlink.com

\begin{abstract}
Goals of work The goal of this study was to examine the relationship between menopausal symptoms, sleep quality, and mood as measured by actigraphy and self-report prior to treatment and at the end of four cycles of chemotherapy in women with breast cancer.

Patients and methods Data on sleep quality (measured using actigraphy and self-report) and mood were collected prior to treatment and 12 weeks later at the end of four cycles of chemotherapy in 69 women with newly diagnosed breast cancer. In addition, each filled out the Greene Climacteric Scale. Based on reported occurrence of menses, participants were categorized post hoc into three menopausal status groups: pre-menopausal before and after chemotherapy (Pre-Pre), pre-menopausal or peri-menopausal before and peri-menopausal after chemotherapy (Pre/Peri-Peri), and post-menopausal before and after chemotherapy (Post-Post). Main results Results suggested that women within the PrePre group evidenced more fragmented sleep with less total sleep time (TST) after chemotherapy compared to baseline. Compared to the other groups, the Pre-Pre group also experienced less TST and more awakenings before and
\end{abstract}

M. B. Rissling $\cdot$ S. Ancoli-Israel

SDSU/UCSD Joint Doctoral Program in Clinical Psychology,

University of California-San Diego,

San Diego, CA, USA

L. Liu $\cdot$ S. Ancoli-Israel $(\square)$

Department of Psychiatry, University of California-San Diego,

9500 Gilman Drive 0733, La Jolla,

San Diego, CA 92093-0733, USA

e-mail: sancoliisrael@ucsd.edu

L. Natarajan $\cdot$ F. He

Department of Family and Preventive Medicine,

University of California-San Diego,

San Diego, CA, USA after chemotherapy. Although the Pre/Peri-Peri group evidenced a greater increase in vasomotor symptoms after chemotherapy, there was no relationship with sleep. All groups evidenced more depressive symptoms after chemotherapy, but depression was not related to measures of sleep.

Conclusions Contrary to the study hypothesis, these results suggest that women who are pre-menopausal or having regular menses before and after four cycles of chemotherapy have worse sleep following chemotherapy. Those women who maintain or become peri-menopausal (irregular menses) experience an increase in climacteric symptoms but do not experience an associated worsening of sleep. These results are preliminary and more research is necessary to further explain these findings.

Keywords Chemotherapy · Menopause - Actigraphy · Sleep quality Breast cancer

\section{Introduction}

Complaints of insomnia and poor sleep are common in women going through natural menopause [1]. It is believed that the primary predictor of disturbed sleep in peri-menopausal women is the presence of vasomotor symptoms, i.e., hot flashes [2-4].

Insomnia and poor sleep are two of the most common symptoms reported by women with breast cancer, with over $50 \%$ of patients complaining of difficulty sleeping before, during, and after chemotherapy [5]. One significant sequelae of chemotherapy is acute ovarian failure which results in either permanent or temporary amenorrhea as well as a sudden onset of the symptoms of menopause [6-9]. 
Earlier detection of breast cancer has lead to an increasingly young population of women being treated with chemotherapy, and thus younger women are going through peri-menopause, i.e., experiencing symptoms of menopause, including symptoms of insomnia. Hot flashes in women with breast cancer are associated with poorer subjective sleep quality $[10,11]$ and thought to contribute to poorer, less consolidated sleep by increasing awakenings and sleep stage changes [3]. Yet, little is known about whether the poor sleep in women undergoing chemotherapy is a result of the concurrent menopausal symptoms.

As part of a larger study on the relationship between sleep and fatigue in women with breast cancer, the current study examined the effects of menopausal symptoms on sleep quality during chemotherapy. Participants were divided post hoc into three menopausal groups based on their self-reported occurrence of menses before and after chemotherapy. For the purposes of this study, sleep quality was defined as the maintenance or change of nighttime sleep characteristics from before and after chemotherapy using both objective and subjective measures. The hypothesis was that women experiencing peri-menopause (defined as irregular menses) at either point during chemotherapy would have worse sleep quality at the end of treatment than pre- or post-menopausal women.

\section{Methods}

Patients

Sixty-nine women diagnosed with stage I-III breast cancer and referred for at least four cycles of adjuvant (chemotherapy after surgery) or neo-adjuvant (chemotherapy before surgery) anthracycline-based chemotherapy were studied (see Fig. 1). Potential participants were recruited from the Rebecca and John Moores University of California-San Diego (UCSD) Cancer Center. Exclusion criteria included women undergoing bone marrow transplants, metastatic breast cancer, confounding underlying medical illnesses such as renal failure or significant preexisting anemia, or other physical or psychological impairments which would limit participation. The study was approved by both the UCSD Human Research Protection Program and the San Diego State University Human Research Protection Program.

\section{Study design}

Most data were collected at seven time points: prior to chemotherapy, during the three weeks of cycle 1, and during the three weeks of cycle 4 chemotherapy. Menopausal symptoms and status were collected only prior to

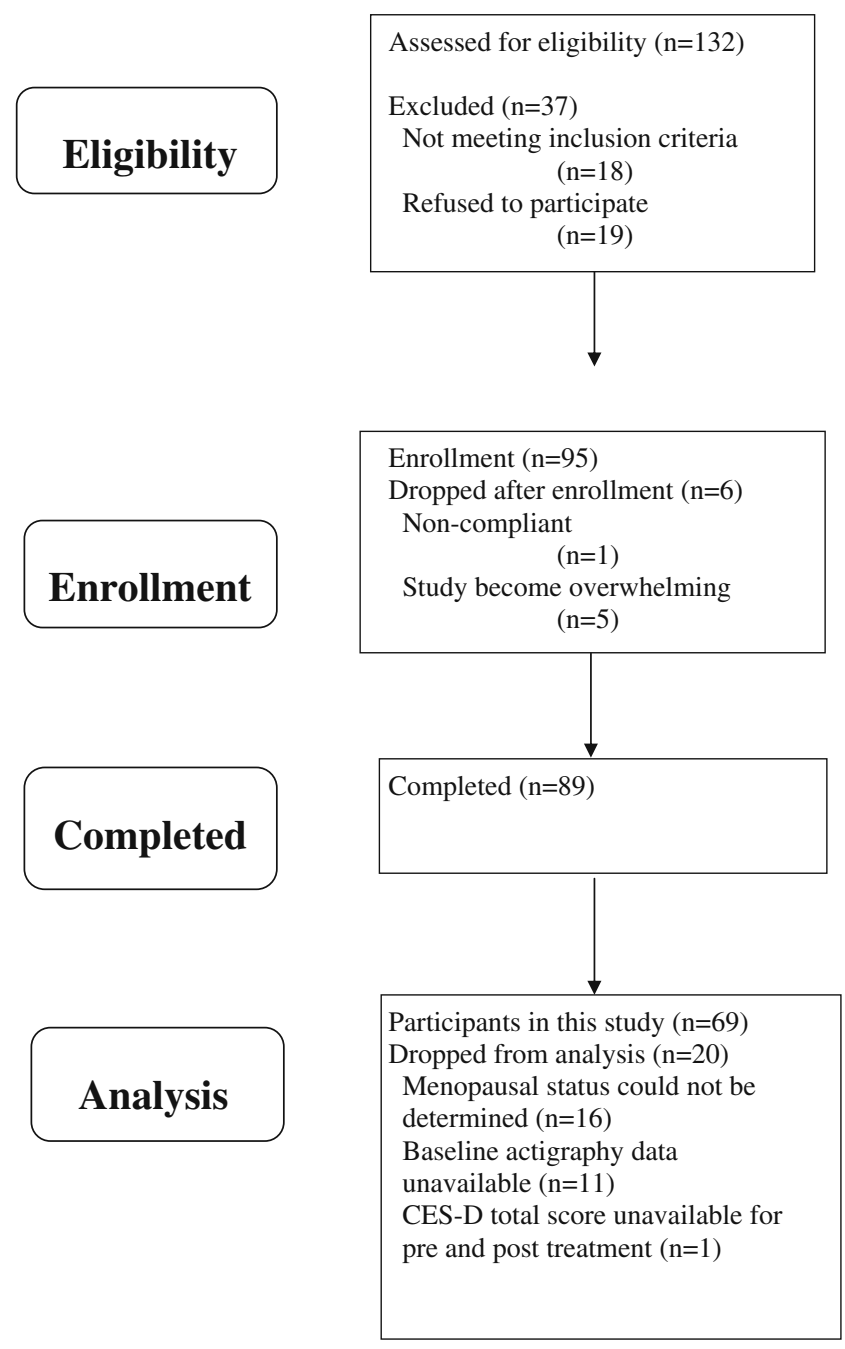

Fig. 1 Screening and enrollment flowchart

chemotherapy and during the recovery or third week of cycle 4 of chemotherapy (end of fourth cycle of treatment). Therefore, only data collected at those two time points were analyzed. Each chemotherapy cycle was three weeks in duration; therefore, the interval from baseline to the end of treatment was 12 weeks. At the start of the study, body weight and height were measured as were general demographics. Body mass index was calculated (in $\mathrm{kg} / \mathrm{m}^{2}$ ).

Women were divided post hoc into three groups based on self-report of the occurrence of menses both prior to chemotherapy and during the recovery week of cycle 4 chemotherapy: group 1, called Pre-Pre $(N=12)$, was composed of women who reported regular menstrual cycles both before and after chemotherapy, group 2, called Pre/ Peri-Peri $(N=21)$, was composed of women who reported irregular menses after chemotherapy but had reported either regular or irregular menses before chemotherapy (combined due to small group size), and group 3, called Post-Post $(N=36)$, was composed of women with amenorrhea before 
and after chemotherapy, including women that were posthysterectomy and/or post-oophorectomy.

\section{Sleep measurements}

(a) Wrist actigraph. The Actillume II actigraph (Ambulatory Monitoring, Inc., Ardsley, NY, USA) was worn by each patient for 72 consecutive hours ( 3 days and 3 nights) at all seven time points. The Actillume II contains a piezoelectric linear accelerometer, a microprocessor, $32 \mathrm{~K}$-byte random access memory, and associated circuitry for the purpose of recording intensity and frequency of movement. The orientation and sensitivity of the accelerometer are optimized for highly effective sleep-wake inference from wrist activity [12-14]. Actigraphic records were scored with Action 3 (Ambulatory Monitoring, Inc., Ardsley, NY, USA) for total sleep time (TST) at night, wake after sleep onset (WASO), and sleep percentage (\%). Sleep onset latency, or time to fall asleep, has been found to be inaccurate when using actigraphy and is therefore not included as a parameter. Sleep percentage is defined as the percent time asleep after sleep onset and does not include the less reliable parameter of sleep onset latency [15]. The number (NWAK) and mean duration of nighttime awakenings and sleep bouts were extracted from the scored actigraphic records using $\mathrm{SAS}^{\circledR}$ software, version 9.1.3 [16]. A daily sleep diary was used to validate time in bed and time awake.

(b) Pittsburgh Sleep Quality Index [PSQI; 17]. The PSQI is a 19-item questionnaire which rates patients' reports of their sleep quality based on ratings of subjective sleep latency, sleep duration, habitual sleep efficiency, sleep disturbances, use of sleep medication, and daytime dysfunction. The global score may range from 0 to 21 with high scores reflecting poor sleep quality. A global score above 5 is generally considered poor sleep.

\section{Other measurements}

(a) Climacteric status. Climacteric status was reported using one of four descriptors: (1) regular menses before/after chemotherapy, (2) irregular menses before/ after chemotherapy, (3) post-menopausal before/after chemotherapy, and (4) post-hysterectomy/oophorectomy before/after chemotherapy.

(b) Greene Climacteric Scale [18]. Climacteric symptomatology was assessed with the Greene Climacteric Scale, a self-report questionnaire that measures a total of 21 physical and psychological symptoms associated with the menopause transition. Each symptom is rated by the respondent according to its severity using a four-point scale: not at all (0), a little (1), quite a bit (2), and extremely (3). Items $1-11$ address anxiety (items 1-6) and depression (items 7-11); examples include 'difficulty in sleeping' and 'feeling unhappy or depressed.' The anxiety and depression item scores are combined to create a psychological subscale score which may range from 0 to 33 . Physical or somatic symptoms such as headaches and muscle and joint pain are measured by items $12-18$; the range for the somatic subscale is $0-21$. Vasomotor symptoms (hot flushes, sweating at night) are assessed by items 19 and 20 and comprise the vasomotor subscale, the range for this subscale is 0 to 6 . Item 21 is a singleitem probe for loss of interest in sex. The total score may range from 0 to 63 .

(c) Center for Epidemiologic Studies Depression Scale [CES-D; 19]. The CES-D is a self-report questionnaire that measures a total of 20 cognitive, affective, behavioral, and somatic symptoms of clinical depression and can range from 0 to 60 , with higher scores indicating greater distress. The total score is comprised of interpersonal, positive, and negative affect subscale scores. The mean score for patients with a diagnosis of clinical depression is 39 , although a score of 16 or above is considered to be clinically significant for distress related to depressive symptoms [20].

\section{Statistical analysis}

A $3 \times 2$ group (Pre-Pre, Pre/Peri-Peri, and Post-Post) by time (baseline and the recovery week of cycle 4) linear mixed model was performed using $\mathrm{R}$, version 2.10.0 (www. rproject.org) on seven dependent objective sleep quality variables collected with actigraphy: TST, \% sleep, WASO, NWAK, mean duration of awakenings, number of sleep bouts, and mean duration of sleep bouts at night. Nighttime awakenings (WASO and NWAK) were defined as a period of wakefulness between two periods of sleep (including sleep onset); sleep bouts were defined as a period of sleep between two periods of wakefulness (including sleep offset). Together, these measurements have been used as an index of sleep consolidation [12]. The largest group, Post-Post, was used as the reference group for all linear mixed-model analyses.

Group differences in demographic, medical, and background characteristics were assessed with $3 \times 2$ linear mixed model for continuous variables and Fisher's exact test for categorical variables.

To probe for statistically significant group differences on the subscale scores and total or global score of the Greene 
Climacteric Scale, the CES-D, and the PSQI, $3 \times 2$ linear mixed-model analyses were conducted. The CES-D total score was included as a covariate in the linear mixed-model analyses of the actigraphy and PSQI variables to control for a possible confounding effect of mood on the subjective and objective sleep variables.

\section{Results}

\section{Demographics}

Complete data from 69 women were included in the analyses (see Table 1). The groups were significantly different in mean age with the Pre-Pre group being the youngest and the Post-Post group the oldest $(P<0.001)$. All three groups were similar in BMI, marital status, income, education, occupation, race, and ethnicity $(P>0.05)$. Cancer stage was also similar across groups $(P>0.05$, Fisher's exact test). As measured by BMI, a majority $(65.4 \%)$ of women in this sample were either overweight $(32.1 \%$; $\mathrm{BMI}=25-29.9)$ or obese $(33.3 \%$; $\mathrm{BMI}=30$ or above).

\section{Sleep}

The mean actigraphic sleep variables for each group are shown in Table 2. There was a significant group-by-time interaction for TST, WASO, \% sleep, duration of wake and sleep bouts, and NWAK. indicating that changes in these
Table 1 Demographics by menopausal status

\begin{tabular}{|c|c|c|c|}
\hline Group & Pre-Pre, $N=12$ & Pre/Peri-Peri, $N=21$ & Post-Post ${ }^{\mathrm{a}}, N=36$ \\
\hline Mean age, years (SD) & $41.6(1.3)$ & $45.6(1.5)$ & $57.9(1.3)^{\mathrm{b}}$ \\
\hline Age range years & $34-49$ & $34-61$ & $43-79$ \\
\hline Mean body mass index (SD) & $30.2(2.6)$ & $26.9(1.3)$ & $29.9(1.1)$ \\
\hline \multicolumn{4}{|l|}{ Ethnicity, $N(\%)$} \\
\hline Caucasian & $7(58.3)$ & $16(76.2)$ & $28(77.8)$ \\
\hline African American & $2(16.7)$ & $0(0.0)$ & $2(5.6)$ \\
\hline Hispanic & $0(0.0)$ & $1(4.8)$ & $2(5.6)$ \\
\hline Asian & $1(8.3)$ & $2(9.5)$ & $2(5.6)$ \\
\hline Native American & $1(8.3)$ & $1(4.8)$ & $0(0.0)$ \\
\hline Other & $1(8.3)$ & $0(0.0)$ & $2(5.6)$ \\
\hline Unknown & $0(0.0)$ & $1(4.8)$ & $0(0.0)$ \\
\hline \multicolumn{4}{|l|}{ Income, $N(\%)$} \\
\hline$<\$ 30,000$ & $0(0.0)$ & $3(14.3)$ & $8(22.3)$ \\
\hline$\geq \$ 30,000$ & $11(91.7)$ & $16(76.2)$ & $24(66.7)$ \\
\hline Refused to answer & $1(8.3)$ & $2(9.5)$ & $4(11.1)$ \\
\hline \multicolumn{4}{|l|}{ Education, $N(\%)$} \\
\hline Some high school or less & $1(8.3)$ & $1(4.8)$ & $0(0.0)$ \\
\hline Completed high school & $1(8.3)$ & $5(23.8)$ & $10(27.8)$ \\
\hline Some college & $4(33.3)$ & $4(14.3)$ & $11(30.6)$ \\
\hline Completed college & $6(50.0)$ & $12(57.1)$ & $15(41.7)$ \\
\hline \multicolumn{4}{|l|}{ Marital status, $N(\%)$} \\
\hline Never married & $3(25.0)$ & $2(9.5)$ & $4(11.1)$ \\
\hline Divorced & $1(8.3)$ & $0(0.0)$ & $11(30.6)$ \\
\hline Separated & $1(8.3)$ & $1(4.8)$ & $0(0.0)$ \\
\hline Widowed & $0(0.0)$ & $0(0.0)$ & $1(2.8)$ \\
\hline Married & $7(58.3)$ & $18(85.7)$ & $20(55.6)$ \\
\hline \multicolumn{4}{|l|}{ Cancer stage, $N(\%)$} \\
\hline I & $3(25.0)$ & $5(23.8)$ & $11(30.6)$ \\
\hline II & $6(50.0)$ & $12(57.1)$ & $13(36.1)$ \\
\hline III & $2(16.7)$ & $3(14.3)$ & $5(13.9)$ \\
\hline III-A & $0(0.0)$ & $1(4.8)$ & $4(11.1)$ \\
\hline Unknown & $1(8.3)$ & $0(0.0)$ & $0(0.0)$ \\
\hline
\end{tabular}


Table 2 Mean (SD) nighttime sleep as measured by actigraphy by menopausal status at baseline and end of treatment

\begin{tabular}{|c|c|c|c|c|c|c|}
\hline \multirow{2}{*}{$\begin{array}{l}\text { Group } \\
\text { Sleep variables }\end{array}$} & \multicolumn{2}{|l|}{ Pre-Pre, $N=12$} & \multicolumn{2}{|c|}{ Pre/Peri-Peri, $N=21$} & \multicolumn{2}{|c|}{ Post-Post ${ }^{\mathrm{a}}, N=36$} \\
\hline & Baseline & $\begin{array}{l}\text { End of } \\
\text { treatment }\end{array}$ & Baseline & $\begin{array}{l}\text { End of } \\
\text { treatment }\end{array}$ & Baseline & $\begin{array}{l}\text { End of } \\
\text { treatment }\end{array}$ \\
\hline Total nighttime sleep time (TST; hours) & $6.24(0.40)$ & $5.60(0.46)^{\mathrm{b}}$ & $6.74(0.27)$ & $6.87(0.37)$ & $6.20(0.23)$ & $6.82(0.27)$ \\
\hline Wake after sleep onset (WASO; hours) & $1.79(0.31)$ & $2.67(0.30)^{\mathrm{b}}$ & $2.07(0.20)$ & $2.17(0.20)$ & $2.31(0.26)$ & $2.10(0.25)$ \\
\hline Sleep percentage, $\%$ & $77.7(3.7)$ & $67.3(3.6)^{\mathrm{b}}$ & $76.3(2.3)$ & $75.5(2.4)$ & $73.7(2.7)$ & $77.2(2.3)$ \\
\hline Duration of sleep bouts (minutes) & $32.30(10.27)$ & $18.79(7.63)^{\mathrm{b}}$ & $27.97(6.61)$ & $27.20(10.21)$ & $31.25(4.94)$ & $37.04(6.94)$ \\
\hline Number of sleep bouts & $21.26(3.18)$ & $29.25(3.23)$ & $25.94(3.28)$ & $24.67(2.46)$ & $19.86(1.66)$ & $20.37(2.39)$ \\
\hline Duration of awakenings (minutes) & $5.13(0.44)^{\mathrm{c}}$ & $5.96(0.60)^{\mathrm{c}}$ & $5.34(0.39)^{\mathrm{c}}$ & $5.64(0.54)^{\mathrm{c}}$ & $7.00(0.53)$ & $6.58(0.59)$ \\
\hline Number of awakenings & $21.26(3.18)$ & $29.25(3.23)^{\mathrm{b}}$ & $26.63(3.30)$ & $25.21(2.55)$ & $20.48(1.70)$ & $20.96(2.43)$ \\
\hline
\end{tabular}

CES-D total score used as covariate in the analyses of all variables

${ }^{a}$ Post-menopausal group used as reference for between group comparisons

${ }^{\mathrm{b}} P<0.05$ for group-by-time interaction effect

${ }^{\mathrm{c}} P<0.05$ for group main effect

sleep variables at the end of chemotherapy compared to baseline were significantly different between women in the Pre-Pre menopausal group compared to the Post-Post group (reference group), with the Pre-Pre group exhibiting a decrease in TST $[t(43)=2.29, P=0.03$, mean group difference in change scores $=-1.18 \mathrm{~h}]$, increase in WASO $[t(43)=2.67, P=0.01$, mean group difference in change scores $=1.14 \mathrm{~h}]$, decrease in sleep percentage $[t(43)=2.15$, $P=0.02$, mean group difference in change scores $=-14 \%]$, shorter duration of sleep bouts $[t(43)=1.99, P=0.04$, mean group difference in change scores $=-20.32 \mathrm{~min}]$, and more awakenings $[t(43)=2.04, P=0.04$, mean group difference in change scores $=7.91$ discrete awakenings]. There were no significant differences in these sleep variables for either the Pre/Peri-Peri or Post-Post groups from baseline to end of treatment. Group-by-time interactions for number of sleep bouts and duration of nighttime awakenings were not significant at the 5\% level. However, there was a main effect of group for both the Pre-Pre and Pre/PeriPeri groups for duration of nighttime awakenings, indicating that both groups had shorter duration of awakenings than the Post-Post group before and after chemotherapy [Pre-Pre: $t(66)=2.21, P=0.03$, mean group difference $=-1.89 \mathrm{~min}$; Pre/Peri-Peri: $t(66)=2.39, P=0.02$, mean group difference $=-1.69 \mathrm{~min}]$.

There were no significant associations between these objective sleep variables and age, race, ethnicity, BMI, income, education, occupation, marital status, neo-adjuvant therapy status, or stage of cancer.

The mean PSQI variables for each group are shown in Table 3. With regard to self-reported sleep quality, there was no significant group, time, or group-by-time interaction effect.
Climacteric status and symptomatology

The mean Green Climacteric Scale variables for each group are shown in Table 4 and are comprised of the total score and six subscales: anxiety, depression, psychological (sum of anxiety and depression), somatic, vasomotor, and sex. There was no significant main effect of time for either the anxiety or psychological subscale for any group; however, there was a significant main effect of time for the depression subscale, indicating that depressive symptoms of menopause increased from baseline to end of treatment $[t(60)=2.43, P=0.02$, mean change in score $=$ 1.2]. There was also a significant group-by-time interaction for the vasomotor subscale showing that women within the Pre/Peri-Peri group reported increased vasomotor symptoms at the end of treatment as compared to the Post-Post group $[t(60)=3.03, P=0.004$, mean change in score $=1.7]$. Furthermore, there was a significant group effect for the sex subscale showing that women within the Pre/Peri-Peri group had worse scores on the sex subscale than women in the Post-Post group both before and after chemotherapy $[t(65)=-2.45, P=0.02$, mean difference between groups $=-0.74]$. There was no significant group, time, or group-by-time interaction effect on any of the remaining subscales or the total score.

\section{Depressive symptoms}

The mean CES-D variables for each group are shown in Table 5 and are comprised of the total score and four subscale scores: positive and negative affect, somatic, and interpersonal. Consistent with the Green Climacteric Scale depression subscale, there was a main effect of time for the 
Table 3 Mean (SD) PSQI results by menopausal status at baseline and end of treatment

\begin{tabular}{|c|c|c|c|c|c|c|}
\hline \multirow[t]{2}{*}{ Group } & \multicolumn{2}{|c|}{ Pre-Pre, $N=12$} & \multicolumn{2}{|c|}{ Pre/Peri-Peri, $N=21$} & \multicolumn{2}{|c|}{ Post-Post ${ }^{\mathrm{a}}, N=36$} \\
\hline & Baseline & End of treatment & Baseline & End of treatment & Baseline & End of treatment \\
\hline Global scores & $8.50(3.87)$ & $8.43(3.74)$ & $6.29(3.54)$ & $6.83(4.29)$ & $6.91(3.43)$ & $7.09(3.86)$ \\
\hline \multicolumn{7}{|l|}{ Component scores } \\
\hline Sleep quality & $1.50(0.52)$ & $1.36(0.93)$ & $1.09(0.81)$ & $1.20(0.77)$ & $1.19(0.78)$ & $1.00(0.75)$ \\
\hline Sleep onset latency & $1.00(0.74)$ & $0.93(1.00)$ & $0.91(1.02)$ & $1.05(0.97)$ & $0.89(1.01)$ & $0.83(0.86)$ \\
\hline Sleep duration & $0.92(0.51)$ & $1.14(0.95)$ & $0.62(0.74)$ & $0.60(0.68)$ & $0.86(0.79)$ & $0.69(0.83)$ \\
\hline Habitual sleep & $1.33(1.30)$ & $1.71(1.33)$ & $0.90(1.26)$ & $0.79(1.13)$ & $0.95(1.22)$ & $0.91(1.25)$ \\
\hline \multicolumn{7}{|l|}{ efficiency } \\
\hline Sleep disturbance & $1.58(0.67)$ & $1.29(0.61)$ & $1.45(0.60)$ & $1.55(0.60)$ & $1.65(0.72)$ & $1.46(0.65)$ \\
\hline $\begin{array}{l}\text { Use of sleep } \\
\text { medications }\end{array}$ & $1.17(1.47)$ & $1.00(1.18)$ & $0.77(1.23)$ & $0.75(1.16)$ & $0.89(1.28)$ & $1.14(1.36)$ \\
\hline Daytime dysfunction & $1.00(0.85)$ & $1.00(0.78)$ & $0.77(0.69)$ & $1.00(0.86)$ & $0.62(0.49)$ & $1.14(0.71)$ \\
\hline
\end{tabular}

CES-D total score used as covariate in the analyses of all variables

${ }^{a}$ Post-menopausal group used as reference for between-groups comparisons no significant comparisons at $P<0.05$

CES-D total score, indicating that overall depressive symptoms increased from baseline to the end of treatment for all three groups $[F(1,50)=6.02, P=0.02]$. There was no significant effect for either the interpersonal or negative affect subscales, suggesting no increase or decrease in these subtypes of depressive symptoms from baseline to the end of treatment for all three groups. The Post-Post group evidenced a significant increase in positive affect symptoms of depression from baseline to the end of treatment [positive affect: $t(35)=3.17, P=0.004]$.

Depressive symptoms and sleep

CES-D total scores were not significantly related to the baseline actigraphy or PSQI sleep variables (data not shown). However, CES-D total scores at the end of

Table 4 Mean (SD) Greene Climacteric Scale results by menopausal status at baseline and end of treatment

\begin{tabular}{|c|c|c|c|c|c|c|}
\hline \multirow[t]{2}{*}{ Group } & \multicolumn{2}{|c|}{ Pre-Pre, $N=12$} & \multicolumn{2}{|c|}{ Pre/Peri-Peri, $N=21$} & \multicolumn{2}{|c|}{ Post-Post ${ }^{\mathrm{a}}, N=36$} \\
\hline & Baseline & End of treatment & Baseline & End of treatment & Baseline & End of treatment \\
\hline Total score & $10.31(1.98)$ & $12.92(2.55)$ & $9.17(1.42)$ & 14.27 (2.09) & $10.90(1.26)$ & $12.78(1.77)$ \\
\hline \multicolumn{7}{|l|}{ Subscales } \\
\hline Anxiety & $3.54(0.67)$ & $2.83(0.63)$ & $3.00(0.60)$ & $3.6(0.74)$ & $3.00(0.35)$ & $3.15(0.43)$ \\
\hline Depression & $2.85(0.65)$ & $4.35(0.89)^{\mathrm{b}}$ & $2.77(0.48)$ & $4.25(0.62)^{\mathrm{b}}$ & $2.87(0.39)$ & $4.07(0.52)^{\mathrm{b}}$ \\
\hline Psychological & $6.39(1.16)$ & $6.67(1.31)$ & $5.48(0.91)$ & $7.48(1.27)$ & $5.84(0.71)$ & $7.26(0.94)$ \\
\hline Somatic & $2.39(0.58)$ & $3.17(0.97)$ & $2.73(0.57)$ & $2.95(0.6)$ & $2.38(0.42)$ & $2.48(0.55)$ \\
\hline Vasomotor & $0.62(0.33)$ & $1.93(0.58)$ & $0.83(0.21)$ & $2.7(0.45)^{\mathrm{c}}$ & $1.53(0.27)$ & $1.73(0.33)$ \\
\hline Sex & $0.92(0.31)$ & $1.25(0.41)$ & $0.58(0.15)^{\mathrm{d}}$ & $1.06(0.23)^{\mathrm{d}}$ & $1.23(0.19)$ & $1.35(0.24)$ \\
\hline
\end{tabular}

${ }^{\text {a }}$ Post-menopausal group used as reference for between-groups comparisons

${ }^{\mathrm{b}} P<0.05$ for time main effect

${ }^{\mathrm{c}} P<0.05$ for group-by-time interaction effect

${ }^{\mathrm{d}} P<0.05$ for group main effect 
Table 5 Mean (SD) CES-D results by menopausal status at baseline and end of treatment

\begin{tabular}{|c|c|c|c|c|c|c|}
\hline \multirow[t]{2}{*}{ Group } & \multicolumn{2}{|c|}{ Pre-Pre, $N=12$} & \multicolumn{2}{|c|}{ Pre/Peri-Peri, $N=21$} & \multicolumn{2}{|c|}{ Post-Post ${ }^{\mathrm{a}}, N=36$} \\
\hline & Baseline & End of treatment & Baseline & End of treatment & Baseline & End of treatment \\
\hline Global score & $9.49(4.43)$ & $12.67(9.53)^{\mathrm{b}}$ & $12.65(10.86)$ & $17.88(14.34)^{\mathrm{b}}$ & $11.30(8.83)$ & $14.65(11.15)^{\mathrm{b}}$ \\
\hline \multicolumn{7}{|l|}{ Component scores } \\
\hline Positive affect & $1.91(1.97)$ & $2.89(2.48)$ & $2.40(3.12)$ & $4.44(4.53)$ & $2.21(2.29)$ & $3.77(3.53)_{\mathrm{c}}$ \\
\hline Negative affect & $1.77(1.75)$ & $2.11(2.20)$ & $3.55(3.83)$ & $4.25(3.92)$ & $2.94(3.03)$ & $3.04(2.78)$ \\
\hline Somatic & $4.05(2.78)$ & $5.56(3.71)$ & $5.20(4.85)$ & $6.56(4.98)$ & $4.33(3.67)$ & $5.90(4.50)_{\mathrm{c}}$ \\
\hline Interpersonal & $0.09(0.30)$ & $0.00(0.00)$ & $0.00(0.00)$ & $0.13(0.34)$ & $0.12(0.42)$ & $0.23(0.67)$ \\
\hline
\end{tabular}

${ }^{\text {a }}$ Post-menopausal group used as reference for between-groups comparisons

${ }^{\mathrm{b}} P<0.05$ for time main effect

${ }^{\mathrm{c}} P<0.05$ for group-by-time interaction effect

treatment were related to shorter sleep bouts at the end of treatment $(r=-0.32, P=0.035)$.

\section{Discussion}

This study found that menopausal status before and after four cycles of chemotherapy was associated with poor sleep as measured by wrist actigraphy. Women who were premenopausal both before and after chemotherapy had worse sleep after chemotherapy than before the start of treatment, spending more time awake during the night and evidencing more sleep fragmentation at the end of treatment. Pre-menopausal women also had worse sleep after chemotherapy than women who were either pre-menopausal prior to chemotherapy but evidenced irregular menses after chemotherapy (i.e., peri-menopausal) or were postmenopausal. In terms of sleep fragmentation, pre- and peri-menopausal women had better sleep (i.e., shorter wake bouts or periods of awakening after sleep onset) than postmenopausal women both before and after chemotherapy. Furthermore, the post-menopausal women evidenced no change in their sleep from pre- to post-chemotherapy.

Attempts were made to further characterize the mechanism behind these group differences in objective sleep. Firstly, the experience of somatic climacteric symptoms was consistent with prior research into the effects of chemotherapy; although the post-menopausal initially reported the most vasomotor symptoms before chemotherapy, after chemotherapy the peri-menopausal group experienced the greatest increase and the most vasomotor symptoms as compared to the post-menopausal group. Secondly, the experience of depressive symptoms was consistent with prior research into the effects of chemo- therapy. Self-reported menopausal-related mood symptoms (i.e., depression and anxiety) worsened for all groups after chemotherapy, although this finding appears to be driven by an increase in menopausal-related symptoms of depression.

Although no group met the CES-D criteria for clinical depression, there was a trend for all three groups to have increased overall depressive symptoms at the end of treatment. Therefore, despite a well-established association between depression and sleep disturbance, self-reports of depressive symptoms failed to account for the sleep disturbances found in the pre-menopausal women. Furthermore, overall depressive symptoms did not differ between groups and, with the exception of shortened sleep bouts after chemotherapy, were not associated with objectively measured sleep.

Thirdly, although the objective measures of sleep showed deterioration from before to after chemotherapy in the pre-menopausal group, self-reported sleep quality did not change for any of the three groups. This is contrary to the consistent finding in cancer that subjective measures of sleep quality and daytime dysfunction do get worse from before to after chemotherapy [21]. In the current study, women in all three groups had poor sleep quality as defined by a global score greater than 5 on the PSQI both before and after chemotherapy (see Table 3). In a psychometric study of the PSQI in cancer, Carpenter and Andrykowski suggested that it may be more appropriate to use a cutoff of 8 or higher to describe poor sleep quality in this population [22]. Applying this criterion to the current study, only the pre-menopausal group reported poor sleep quality; this report was consistent both before and after chemotherapy. These findings may suggest a misappraisal of the experience of sleep in the pre-menopausal group before chemotherapy which may have contributed to the worsening of 
sleep after chemotherapy; however, the current study does not provide support for this relationship. Ancoli-Israel, Liu, Marler, Parker, Jones, et al. [23] suggested that objective and subjective sleep measures are often unrelated because reports of sleep time may be overestimated and the time to fall asleep underestimated. Moreover, in the current study, as in previously reported studies, the time frame for the objective and subjective measures was different, as the PSQI measures sleep over the previous 7 days as compared to 3 days of actigraphic recording.

Older age, female gender, and Caucasian race all have been associated with increased reports of sleep problems $[24,25]$. Participants in this study were predominantly Caucasian $(61 \%)$, but there were no significant differences in race or ethnicity. Not surprisingly, our groups significantly differed in age, with the post-menopausal women being oldest and the pre-menopausal women being youngest. Increasing age and post-menopausal status had been found to predict poor sleep in previous studies, but a similar relationship was not found in this study. However, our study was not designed to characterize aging and sleep per se; as such, our groups have a wide age range and at the outset of the study all of our participants were recently diagnosed with a major illness. It is possible that the poor sleep found in our all our groups was related to the cancer and the chemotherapy and not to aging.

Cancer stage has been considered a predominant precipitating factor in sleep disturbance [26] and was as such considered as a contributing factor. However, there was no significant difference in the number of women with each stage of cancer between the three groups, although the pre-menopausal group demonstrated a higher but nonsignificant incidence of stage III. Stage II was the most predominant cancer stage in all three groups, with the postmenopausal group demonstrating a higher but nonsignificant incidence of stage I cancer. Furthermore, cancer stage was not associated with any objective sleep variable. Additionally, our sample was comprised predominantly by women with adjuvant chemotherapy status and such status was not associated with any objective sleep variable; moreover, a larger study that included our sample had already found no association between neoadjuvant or adjuvant chemotherapy status and sleep [23].

The limitations of this study included a lack of non-cancer comparison group, a small pre-menopausal group, uneven group sizes (but likely representative of the frequency of menopausal status in women diagnosed with breast cancer and treated with four cycles of chemotherapy), no follow-up after the end of cycle 4 of chemotherapy, and no measure of pain, a possible contributor to disturbed sleep [11]. The objective measure of sleep, rest-activity actigraphy, was time-locked to the participants' chemotherapy schedules and may have failed to capture weekday/weekend variation.
Previous studies of population-based menopause have found an association between early peri-menopause and sleepdisordered breathing [27] which may disrupt sleep, but this information is not available in this sample.

Another possible limitation of this study was the lack of an objective measure of menopausal status, such as folliclestimulating hormone level. However, in studies of natural menopause, there is growing evidence that folliclestimulating hormone may be an unreliable diagnostic measure and the onset of irregular menses may be the best stand-alone indicator of peri-menopause [28-30]. A similar inconsistency exists in studies of chemotherapy-related amenorrhea; menopausal status has been determined from a variety of measures, including age, follicle-stimulating hormone level, and the occurrence of menses over periods ranging from 12 months pre-treatment to 5 years posttreatment [6]. The shorter duration (3 months) of this study may have misclassified some women as pre-menopausal that, in a longer study, may have been classified as perimenopausal and vice versa.

Our data demonstrate an increase in climacteric symptoms by the end of treatment for the pre-menopausal group that, while not statistically significant, may indicate the beginning of peri-menopause for women in this group. However, there is a lack of scientific support for clinical cut-points using the Greene Climacteric Scale with respect to menopausal status in either healthy women or women undergoing chemotherapy.

In conclusion, the findings of this study showed that women who are pre-menopausal before and after chemotherapy have worse sleep than other women. These results may suggest that the association between peri-menopause and disturbed sleep is weakened in women being treated with chemotherapy. No one factor or combination of factors explained the differences in sleep quality found between our groups, i.e., neither age, anxiety, cancer stage, depression, race, ethnicity, marital status nor subjective self-report of sleep quality was found to contribute to the poorer sleep found in the pre-menopausal group. These results are preliminary and further studies and replications are needed. In particular, physical or psychological factors such as prior exposure to menopausal symptoms that were not studied may have protected the peri- and post-menopausal group from sleep disruption during chemotherapy or made the pre-menopausal group more vulnerable to the effects of chemotherapy. Nevertheless, in a clinical environment, these results suggest that women who do not experience chemotherapy-related amenorrhea within the first four cycles may experience more sleep disruption during chemotherapy treatment. These women may greatly benefit from early non-pharmacologic interventions such as nutrition counseling, sleep hygiene training, or cognitive behavioral therapy for insomnia [31-34]. 
Acknowledgements Supported by the National Cancer Institute CA85264, National Cancer Institute CA112035, National Institute on Aging AG08415, the UCSD General Clinical Research Center (MO1-RR00827), the Rebecca and John Moores UCSD Cancer Center (National Cancer Institute Core Grant P30 CA-23100), and the Research Service of the Veterans Affairs San Diego Healthcare System.

Open Access This article is distributed under the terms of the Creative Commons Attribution Noncommercial License which permits any noncommercial use, distribution, and reproduction in any medium, provided the original author(s) and source are credited.

\section{References}

1. Hsu HC, Lin MH (2005) Exploring quality of sleep and its related factors among menopausal women. J Nurs Res 13:153164

2. Eichling PS, Sahni J (2005) Menopause related sleep disorders. J Clin Sleep Med 1:291-300

3. Savard J, Davidson JR, Ivers H, Quesnel C, Rioux D, Dupere V, Lasnier M, Simard S, Morin CM (2004) The association between nocturnal hot flashes and sleep in breast cancer survivors. J Pain Symptom Manage 27:513-522

4. Kravitz HM, Ganz PA, Bromberger J, Powell LH, Sutton-Tyrrell K, Meyer PM (2003) Sleep difficulty in women at midlife: a community survey of sleep and the menopausal transition. Menopause 10:19-28

5. Savard J, Morin CM (2001) Insomnia in the context of cancer: a review of a neglected problem. J Clin Oncol 19:895-908

6. Bines J, Oleske DM, Cobleigh MA (1996) Ovarian function in premenopausal women treated with adjuvant chemotherapy for breast cancer. J Clin Oncol 14:1718-1729

7. Collichio F, Pandya K (1994) Amenorrhea following chemotherapy for breast cancer: effect on disease-free survival. Oncology (Williston Park) 8:45-52

8. DelMastro L, Venturini M, Sertoli MR, Rosso R (1997) Amenorrhea induced by adjuvant chemotherapy in early breast cancer patients: prognostic role and clinical implications. Breast Cancer Res Treat 43:183-190

9. Goodwin PJ, Ennis M, Pritchard KI, Trudeau M, Hood N (1999) Risk of menopause during the first year after breast cancer diagnosis. J Clin Oncol 17:2365-2370

10. Polo-Kantola P (2007) Sleep and menopause. Womens Health 3:99-106

11. Bardwell WA, Profant J, Casden DR, Dimsdale JE, Ancoli-Israel S, Natarajan L, Rock CL, Pierce JP (2008) The relative importance of specific risk factors for insomnia in women treated for early-stage breast cancer. Psychooncology 17:9-18

12. Ancoli-Israel S, Clopton P, Klauber MR, Fell R, Mason W (1997) Use of wrist activity for monitoring sleep/wake in demented nursing-home patients. Sleep 20:24-27

13. Ancoli-Israel S, Cole R, Alessi C, Chambers M, Moorcroft W, Pollak CP (2003) The role of actigraphy in the study of sleep and circadian rhythms. Sleep 26:342-392

14. Cole RJ, Kripke DF, Gruen W, Mullaney DJ, Gillin JC (1992) Automatic sleep/wake identification from wrist activity. Sleep $15: 461-469$
15. Lichstein KL, Stone KC, Donaldson J, Nau SD, Soeffing JP, Murray D, Lester KW, Aguillard RN (2006) Actigraphy validation with insomnia. Sleep 29:232-239

16. SAS Institute Inc. (2000-2004). SAS 9.1.3 help and documentation. SAS Institute Inc., Cary, NC

17. Buysse DJ, Reynolds CF 3rd, Monk TH, Berman SR, Kupfer DJ (1989) The Pittsburgh Sleep Quality Index: a new instrument for psychiatric practice and research. Psychiatry Res 28:193-213

18. Greene JG (1998) Constructing a standard climacteric scale. Maturitas 29:25-31

19. Devins GM, Orme CM, Costello CG, Binik YM, Frizzell B, Stam HJ, Pullin WM (1988) Measuring depressive symptoms in illness populations: psychometric properties of the Center for Epidemiologic Studies Depression (CES-D) Scale. Psychol Health 2:139-156

20. Radloff LS (1977) The CES-D scale: a self-report depression scale for research in the general population. Applied Psychol Measurement 1:385-401

21. Ancoli-Israel S, Moore PJ, Jones V (2001) The relationship between fatigue and sleep in cancer patients: a review. Eur J Cancer Care (Engl) 10:245-255

22. Carpenter JS, Andrykowski MA (1998) Psychometric evaluation of the Pittsburgh Sleep Quality Index. J Psychosom Res 45:5-13

23. Ancoli-Israel S, Liu L, Marler MR, Parker BA, Jones V, Sadler GR, Dimsdale J, Cohen-Zion M, Fiorentino L (2006) Fatigue, sleep, and circadian rhythms prior to chemotherapy for breast cancer. Support Care Cancer 14:201-209

24. Blazer DG, Hays JC, Foley DJ (1995) Sleep complaints in older adults: a racial comparison. J Gerontol A Biol Sci Med Sci 50: M280-284

25. Rediehs MH, Reis JS, Creason NS (1990) Sleep in old age: focus on gender differences. Sleep 13:410-424

26. Savard J, Simard S, Blanchet J, Ivers H, Morin CM (2001) Prevalence, clinical characteristics, and risk factors for insomnia in the context of breast cancer. Sleep 24:583-590

27. Young T, Finn L, Austin D, Peterson A (2003) Menopausal status and sleep-disordered breathing in the Wisconsin Sleep Cohort Study. Am J Respir Crit Care Med 167:1181-1185

28. Brambilla DJ, McKinlay SM, Johannes CB (1994) Defining the perimenopause for application in epidemiologic investigations. Am J Epidemiol 140:1091-1095

29. Burger HG, Dudley EC, Robertson DM, Dennerstein L (2002) Hormonal changes in the menopause transition. Recent Prog Horm Res 57:257-275

30. Stellato RK, Crawford SL, McKinlay SM, Longcope C (1998) Can follicle-stimulating hormone be used to define menopausal status? Endocr Pract 4:137-141

31. Berger AM, VonEssen S, Kuhn BR, Piper BF, Farr L, Agrawal S, Lynch JC, Higginbotham P (2002) Feasibility of a sleep intervention during adjuvant breast cancer chemotherapy 29:1431-1441

32. Fiorentino L, Ancoli-Israel S (2006) Insomnia and its treatment in women with breast cancer. Sleep Med Rev 10:419-429

33. Shapiro SL, Bootzin RR, Figueredo AJ, Lopez AM, Schwartz GE (2003) The efficacy of mindfulness-based stress reduction in the treatment of sleep disturbance in women with breast cancer-an exploratory study. J Psychosom Res 54:85-92

34. Ahlberg K, Ekman T, Gaston-Johansson F, Mock V (2003) Assessment and management of cancer-related fatigue in adults. The Lancet 362:640-650 